\title{
MODALIDADES SÍNCRONAS E ASSÍNCRONAS NO ENSINO DA RADIOLOGIA DO SISTEMA NERVOSO: ESTILOS E RESULTADOS DE APRENDIZAGEM
}

\author{
(SYNCHRONOUS AND ASYNCHRONOUS TEACHING METHODS OF NERVOUS SYSTEM \\ IMAGING: STYLES AND LEARNING OUTCOMES)
}

Maria Margarida do Carmo Pinto Ribeiro

Escola Superior de Tecnologia da Saúde de Lisboa (Portugal)

Filomena Isabel Gonçalves Batalha

Hospitalar Lisboa Central -Hospital de Santa Marta (Portugal)

Rosa Joana dos Santos Patrocínio

Hospital de Curry Cabral (Portugal)

\section{RESUMO}

Lidar com as problemáticas da saúde, implica um domínio dos processos cognitivos (raciocínio, resolução de problemas e tomada de decisão) e de desempenhos práticos, o que obriga a afectação de um conjunto de atitudes e comportamentos específicos.

Este estudo, implementou e avaliou o impacto de experiências pedagógicas desenvolvidas com os estudantes da unidade curricular Radiologia do Sistema Nervoso (RSN) da Escola Superior de Tecnologia da Saúde de Lisboa (ESTeSL). Aplicaram-se metodologias de ensino mistas (presenciais e virtuais) utilizadas na leccionação teórica e prática no ano curricular 2008/2009. Para a avaliação do perfil de aprendizagem dos estudantes foi aplicado o método de Honey \& Munford e para a avaliação e monitorização dos conhecimentos aplicaram-se check list baseadas nos conteúdos programáticos. A monitorização das ferramentas da plataforma moodle complementaram a restante informação.

Verificou-se uma progressão de aprendizagem positiva para um grupo de estudantes maioritariamente do estilo reflexivo (média=10,6 estudantes).

As conclusões apontaram para um impacto positivo quanto à aplicação das metodologias híbridas com maior índice de sucesso para a metodologia assíncrona. Verificou-se também mais flexibilidade no acesso aos conteúdos porém com algumas limitações tais como residência inicial por parte dos estudantes, maior carga de trabalho para os docentes, falta de terminais para acesso à plataforma $\mathrm{e}$ pouca experiência de todos os envolvidos no domínio e manipulação da plataforma. 
Palavras-chave: ensino clássico, ensino à distância, aprendizagem, tecnologia web; radiologia, e-learning.

\begin{abstract}
This study focused on the role of cognitive processes (reasoning, problem solving and decision making) and performance practice in the formation of attitudes and behaviours relating to health issues. It was conducted to evaluate the effects of pedagogical experiences on students who participated in the course in radiography in the Nervous System Imaging Unit (RSN) of the Lisbon Health School of Technology. Mixed (face-to-face and virtual) teaching methodologies were used in theory and practice sessions. Honey and Munford's method was used to evaluate the learning profile of students. To monitor and evaluate students' knowledge acquisition, check lists based on program topics were applied. Other information was supplied through the learning platform of Moodle. The student group with mostly a reflective learning style increased their knowledge. The asynchronous method was shown to produce a higher success rate and more flexibility in accessing content but also registered some limitations such as resistance by students, increased workload for teachers, lack of access to the platform and inexperience of all involved in handling the platform.
\end{abstract}

Keywords: classical education, distance learning, learning, web technology, radiology, e-learning.

Ao longo das últimas décadas com a consolidação das áreas de conhecimento científico e tecnológico, as instituições de Ensino Superior Politécnico têm desenvolvido formas de ajuste das suas formações ao aperfeiçoamento dos perfis profissionais dos seus educandos.

É imposta, no entanto, em consequência das políticas gestionárias dos últimos governos a introdução de estratégias educativas que promovam a qualidade do ensino adaptadas à racionalização de meios e recursos sob a égide da expansão dos interesses científicos tecnológicos e culturais.

Estas intenções foram expressamente realçadas nas "Grandes Opções do Plano 2005-2009" - lei n ${ }^{0}$. 52/2006, onde se destacam o apelo ao lançamento de iniciativas tendentes à qualificação e formação profissional e ao desenvolvimento científico e tecnológico como antecedentes do incremento de uma escala de valor nacional.

Segundo o grupo de peritos da OCDE, no seu relatório de 7 de Março de 2008, foi reconhecida a dinâmica das reformas empreendidas em Portugal neste domínio e foi salientado o papel dos Institutos Superiores Politécnicos nas seguintes palavras: "Greater access is focused in the polytechnics whose role has been clarified and 
study programs had been made more relevant to the labour market. There has been significant growth in the number of CET programs and enrolment in them".

Posteriormente, vários países do espaço europeu em texto compilado no documento "The Futur of Science and Tecnhology in Europe", realçaram o investimento na internacionalização, na qualidade e na avaliação, prosseguindo uma tendência convergente com as melhores práticas internacionais.

O documento de 2009 (44-45) refere que as metodologias de ensino e aprendizagem estão ainda bastante aquém da utilização plena das Tecnologias de Informação e Comunicação (TIC). Foi referido expressamente um estudo feito no Reino Unido, onde em 122 instituições de ensino avaliadas pelo Observatório do Ensino Superior se verificou que apenas em 6,6\% essa utilização era feita com finalidades pedagógicas, sendo que as outras restantes apenas as utilizavam como recurso administrativo.

A reunião dos 46 países em Lovaina em 29 de Abril de 2009, cujo principal objectivo foi a avaliação das políticas educativas decorrentes do processo de Bolonha, classificou a criação de sinergias nas modalidades de ensino entre países fronteiriços como factor crítico do sucesso deste desafio e veio a reforçar tudo o anteriormente invocado.

Considerando ainda os eixos prioritários definidos nas metas para 2009, no Programa "Compromisso com a Ciência" (2006), no qual se realça o reforço das competências científicas e técnicas preferencialmente da Cultura Científica e Tecnológica, este projecto ambiciona convergir com a reforma de Bolonha, com as orientações do executivo e com as necessidades emergentes detectadas na leccionação do Curso Superior de Radiologia da Escola Superior de Tecnologia da Saúde de Lisboa.

Parafraseando Copetto (2006) sobre o Ensino Superior Português: "o resto do mundo não vai ficar parado à espera que Portugal atinja padrões europeus”.

\section{JUSTIFICAÇÃO}

As recentes abordagens por b-learning (Blended Learning) têm tomado progressivamente o espaço das técnicas clássicas de ensino. Elas visam entre outras finalidades fomentar a interacção e a colaboração entre docentes e discentes bem como potenciar o uso das tecnologias web na criação de novos contextos de 
aprendizagem. Numa convergência do ensino On-line, virtual ou assíncrono e o Face-a-Face, presencial ou síncrono e indo ainda ao encontro da filosofia do modelo educacional de Bolonha, estas metodologias consistem na combinação de vários métodos para a flexibilização dos contextos de aprendizagem.

Pretende-se criar ao estudante a maior variedade de situações em cenário académico, simulando o mais possível os desafios dos contextos de prática clínica real para potenciar um crescente na maturidade profissional. Admite-se que práticas inovadoras em que coexistem técnicas e metodologias de ensino e aprendizagem utilizadas sob a forma de estratégias educacionais mistas e cruzadas em integração progressiva, possam envolver e motivar os estudantes.

Assim, este estudo, implementou e avaliou o impacto de experiências pedagógicas desenvolvidas com os estudantes da unidade curricular Radiologia do Sistema Nervoso (RSN) da Escola Superior de Tecnologia da Saúde de Lisboa (ESTeSL), sendo esta unidade curricular (UC) parte do plano curricular da Licenciatura em Radiologia e com 5 ECTS. Aplicaram-se metodologias de ensino mistas, utilizadas na leccionação teórica e prática no ano curricular 2008/2009, à luz das referências de Bolonha, com base nas recomendações da OCDE e apoiadas na reforma do ensino superior Politécnico o qual se pretende elevar ao nível da excelência.

Pensa-se que as metodologias adoptadas, se bem sucedidas, depois de testadas e consolidadas pela experiência poderão ser alargadas a outras UC (s) da ESTeSL podendo também constituir um modelo para outras instituições de Ensino Superior.

\section{Objectivos gerais:}

- Contribuição para a concretização do modelo educacional de Bolonha no Curso Superior de Radiologia.

- Rentabilização dos recursos tecnológicos existentes e redução de custos evitando a contratação de mais docentes substituindo algumas actividades pela intermediação das TIC.

- Dotar os estudantes de maior responsabilização no seu processo de aprendizagem através do desenvolvimento da concepção, inovação e análise crítica.

- Indução dos estudantes para a construção da compreensão performativa, tornando-os mais activos, dinâmicos, exigentes esistemáticos no desenvolvimento do trabalho. 
- Desenvolvimento de ambientes de verdadeira dinâmica de grupo em aprendizagem cooperativa fazendo-lhes entender que em saúde não se deve decidir sozinho.

- Aproximação dos estudantes aos contextos de ensino na Europeu.

- Promoção do desenvolvimento de estratégias de moderação sustentáveis na aprendizagem on-line.

\section{DESCRIÇÃO METODOLÓGICA}

\section{Determinação de estilos de aprendizagem}

Foi utilizada escala de Honey \& Munford a qual classifica os processos cognitivos e de raciocínio conducentes à aprendizagem segundo uma escala gradativa.

O instrumento consta de 80 proposições de resposta dicotómica: verdadeiro e falso às quais o estudante responderá de acordo com a sua identificação para com o comportamento/atitude/opinião expressos.

Cada resposta está associada a um estilo de aprendizagem. A determinação do estilo está relacionada com o intervalo de respostas afirmativas para cada um. De acordo com o intervalo de respostas obtido para cada um dos estilos, o estudante obterá uma ponderação dirigida a cada um dos estilos de aprendizagem. (Figura 1).

A classificação dos quatro estilos de aprendizagem é:

- Reflexivo - O estudante afasta-se e observa, é cauteloso utilizando sempre um suporte de retaguarda. Recolhe e analisa os dados com base em experiências. Reflecte bem sobre as conclusões. Usa informação do passado, presente e imediata para permitir perspectivar os novos acontecimentos.

- Teórico - Gere os problemas de uma forma lógica, racional e objectiva. Assimila factos díspares assentes em teorias coerentes.

É por natureza disciplinado, com o objectivo de ajustar as coisas em ordem racional. Apoia-se em pressupostos básicos, princípios, teorias, modelos e sistemas de pensamento. 
- Activo - Mergulha plenamente em novas experiências. O seu lema é desfrute aqui e agora. "Open minded", entusiasmado, flexível. Em primeira instância actua e considera as consequências mais tarde. Procura centrar as actividades em torno de si.

- Pragmático - Tem tendência a dar ideias, teorias e pôr técnicas em prática. Busca novas experiências e soluções. Age com rapidez e confiança, vai directo ao assunto a tratar. Fica impaciente com discussões intermináveis.

\begin{tabular}{|c|c|c|c|c|c|}
\hline Estilos & $\begin{array}{c}\text { Muito } \\
\text { elevado }\end{array}$ & Elevado & Moderado & Baixo & $\begin{array}{c}\text { Muito } \\
\text { baixo }\end{array}$ \\
\hline I-Activo & $13-20$ & $11-12$ & $7-10$ & $4-6$ & $0-3$ \\
\hline II-Reflexivo & $18-20$ & $15-17$ & $12-14$ & $9-11$ & $0-8$ \\
\hline III-Teórico & $16-20$ & $14-15$ & $11-13$ & $8-10$ & $0-7$ \\
\hline IV-Pragmático & $17-20$ & $15-16$ & $12-14$ & $9-11$ & $0-8$ \\
\hline
\end{tabular}

Tabla 1. Grelha para a obtenção da classificação final dos estilos de aprendizagem segundo Honey \& Munford

Dentro das metodologias pedagógicas adoptadas, as sessões práticas contaram com grupos entre 5 e 10 estudantes e foram articuladas de forma a promover a aquisição dos duos de competências: metodológicas e instrumentais, sociais e interpessoais, e cognitivas e sistémicas.

Foram avaliados todos os estudantes inscritos na Unidade Curricular (35 observados). A média de idades foi de 19,5 anos.

Houve inicialmente um momento preparatório no qual o contracto pedagógico foi proposto e analisado servindo depois de guião orientador do projecto. Este foi acolhido pelos estudantes sem qualquer sugestão de alteração.

Depois iniciaram-se os dois módulos de leccionação de conteúdos os quais diferiram na forma de leccionação.

Módulo I (7 semanas): A) Introdução às Neurociências; B) Crânio; C) Técnicas não Convencionais e suas principais aplicações.

Módulo II (7 semanas): D) Medula; E) Intervenção em Neurorradiologia; F) Radiologia do Sistema Nervoso na população pediátrica; G) Emergência em Neurorradiologia. 
Adoptaram-se na leccionação da Unidade Curricular de Radiologia do Sistema Nervoso as seguintes metodologias de ensino e técnicas híbridas:

Modalidades síncronas

- Presencial (em sala de aula utilizando metodologias expositivas, demonstrativas, ilustrativas e exemplificativas)

Modalidades assíncronas

- Técnicas mistas ou blended learning

Para a obtenção de resultados fidedignos no que respeita à avaliação do ensino e aprendizagem desta UC e comparabilidade dos resultados entre os distintos métodos e técnicas aplicados, submeteram-se todos os estudantes ao mesmo tipo de metodologia.

As opções metodológicas foram aplicadas de acordo com a seguinte organização:

\section{Aulas teóricas}

Tipologia de Contacto presencial em Modalidade síncrona pelas técnicas clássicas

Acções pedagógicas:

- Apresentação dos conteúdos teóricos em sala com a frequência de 1 sessão semanal de 2 horas.

Metodologias e recursos: Apresentação expositiva de conteúdos utilizando métodos pedagógicos de Ilustração; exemplificação e demonstração. Orientação de estudo segundo os tópicos programáticos propostos.

Tipologia de Contacto por orientação Tutória em Modalidade Assíncrona:

Acções pedagógicas:

- Disponibilização de conteúdos através da plataforma on-line moodle.

- Apresentação de problemas clínicos. 
- Orientação tutória de trabalhos em estudo independente do estudante.

- Fóruns de discussão e Quiz.

Metodologias e recursos: Discussão de tópicos em fórum; Trabalhos de grupo e trabalho individual; Estudos de casos clínicos; estudo individual; ensaios; relatórios; exames clínicos em contexto real.

\section{Aulas práticas}

Tipologia síncrona em complemento com ambos os módulos I e II

Acções pedagógicas:

- Proposta de tópicos programáticos desencadeando um estímulo dirigido à resposta de cada situação clínica.

Metodologias e recursos: Naárea laboratorial da ESTeSL-apresentação expositiva de materiais pedagógicos (Figura5); Discussão e caracterização das soluções de diagnóstico clínico por imagem (Figura2); Manipulação e processamento de imagens utilizando tecnologia informática - Workstations; (Figura 4) utilização de fantomas antropomórficos para aquisição de imagens radiológicas reais (Figura 3); manipulação de peças anatómicas no teatro anatómico da FCM-UNL (Figuras 6, 7 e 8); hospitais ou outros locais de prática clínica; Estudos de casos clínicos; ensaios; relatórios; exames clínicos em contexto real, manipulação de peças anatómicas. Guião de aula prática. Foram ainda usados os seguintes recursos: Teatching files; Grelhas para análise de artigos científicos; Exercícios práticos em Anatomia Radiológica; Tabelas de protocolos Técnicos e discussão dos mesmos. 


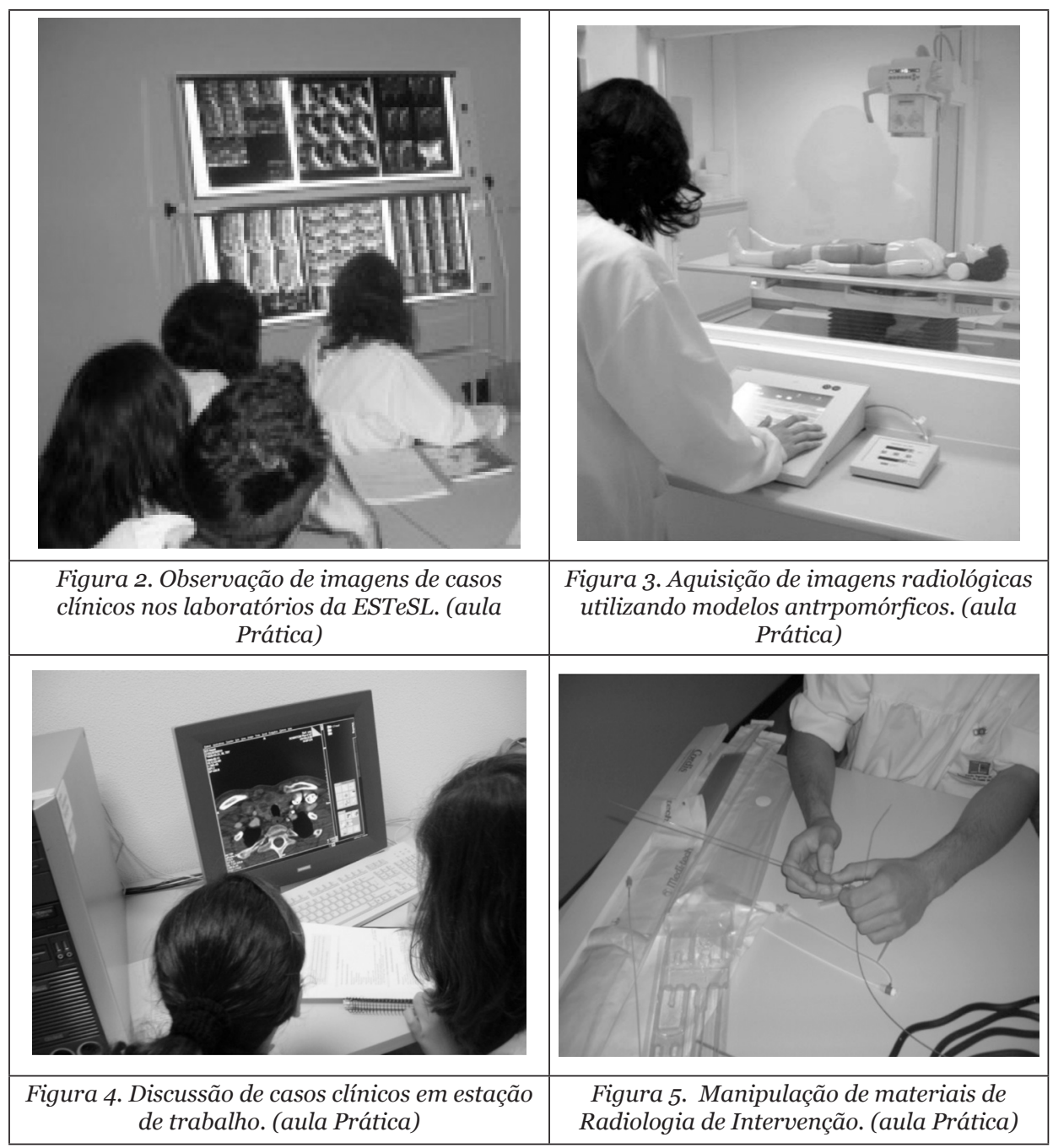



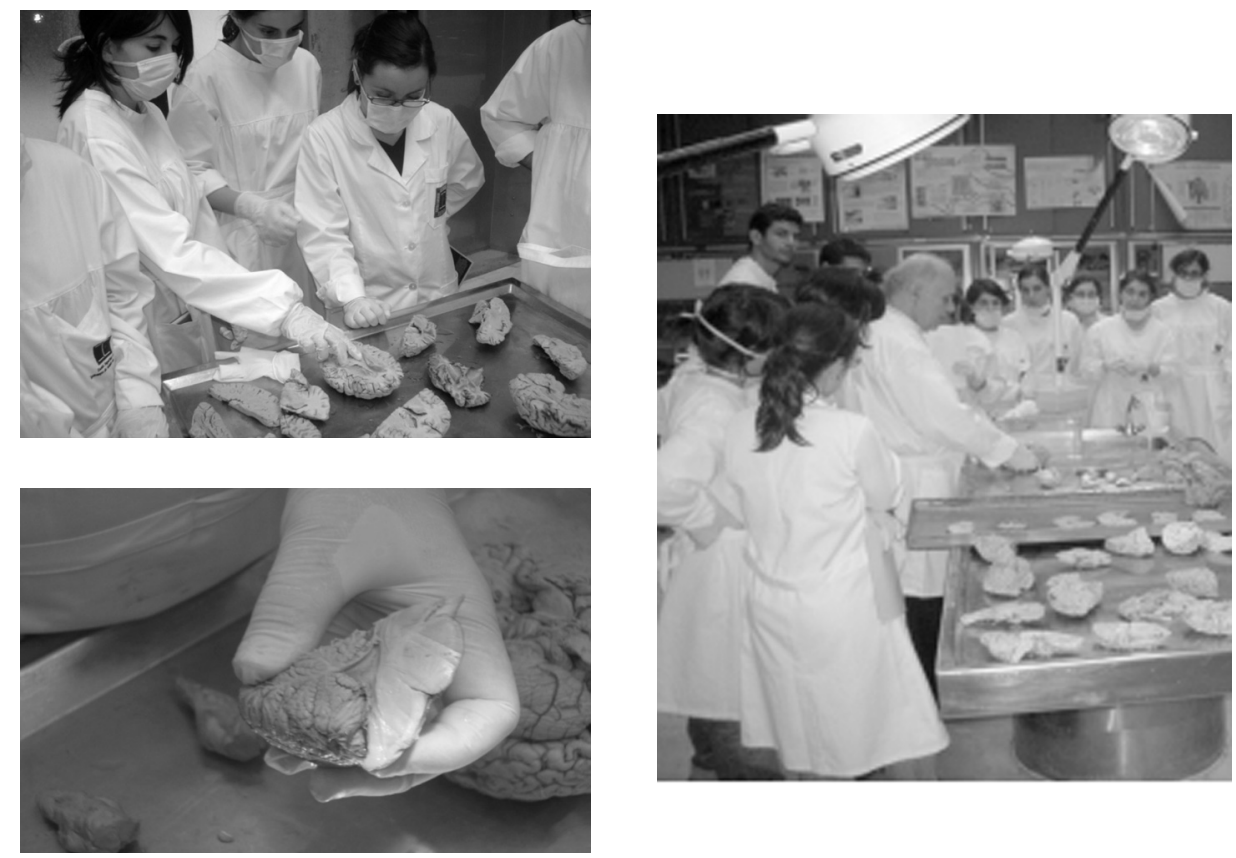

Figuras 6, 7 e 8. Manipulação de peças anatómicas no Teatro Anatómico da Faculdade de Ciências Médicas (aula Prática)

\section{INSTRUMENTOS DE RECOLHA DE DADOS}

Os instrumentos de recolha de dados consideraram-se agrupados segundo avaliação de conhecimentos e monitorização de conhecimentos.

\section{Avaliação:}

- Método de Honey-Mumford para a determinação do perfil de aprendizagem do estudante em activo, reflexivo, teórico ou pragmático.

- Primeira aplicação de uma ficha de diagnóstico de conhecimentos (check list) que versaram temas relativos a matérias do plano curricular.

- Instrumentos de avaliação da UC e respectivos resultados de aprendizagem.

Avaliação escrita individual dividida em duas partes:

Em que a parte 1, foi aplicada através da plataforma moodle. Incluiu 20 respostas de resposta múltipla e questões de resposta dicotómica $-\mathrm{V}$ ou F- , de correcção automática e com uma tentativa de resposta. 
A parte 2, foi aplicada de forma presencial incluindo questões para resposta curta e do tipo de resposta dicotómica V e F.

Foi garantido entre as partes 1 e 2, igual número de questões, foi dado o mesmo tempo para resposta e garantiu-se que o grau de complexidade fosse similar. Esta validação quanto ao grau de dificuldade foi efectuada por dois docentes em sistema double-blind.

Actividades complementares de avaliação também divididas em duas partes:

A parte 1, constou de um trabalho escrito sobre um tema relacionado com os conteúdos leccionados e a parte 2, referiu-se a outras actividades como por exemplo Teatching files; análise de artigos científicos; exercício prático em Anatomia Radiológica; elaboração de protocolos Técnicos e procedimentos; descrição de vídeos relativos a procedimentos e discussão de casos clínicos.

\section{Monitorização:}

- Segunda aplicação de uma ficha de diagnóstico de conhecimentos (check list).

- Instrumentos de avaliação da UC.

- Consulta das ferramentas e estatísticas de participação na plataforma moodle pela avaliação dos trabalhos complementares aos conteúdos leccionados.

Out puts em forma de dados estatísticos e registos gráficos semanais dos relatórios de actividades e de participação por actividade e por estudante como resposta aos trabalhos propostos, bem como o registo de acesso, consultas, contribuições, gráficos de processo através da interface moodle, e sua avaliação.

\section{Resultados}

Da avaliação do perfil dos estudantes (Gráfico1) resulta que para uma taxa de resposta de $94 \%$, a média dos estilos de aprendizagem situou-se maioritariamente no estilo reflexivo com um número de cerca de 11 estudantes seguindo-se o estilo teórico com 8. Os estilos Activo e Pragmático foram os menos verificados apenas com aproximadamente 6 estudantes cada. 


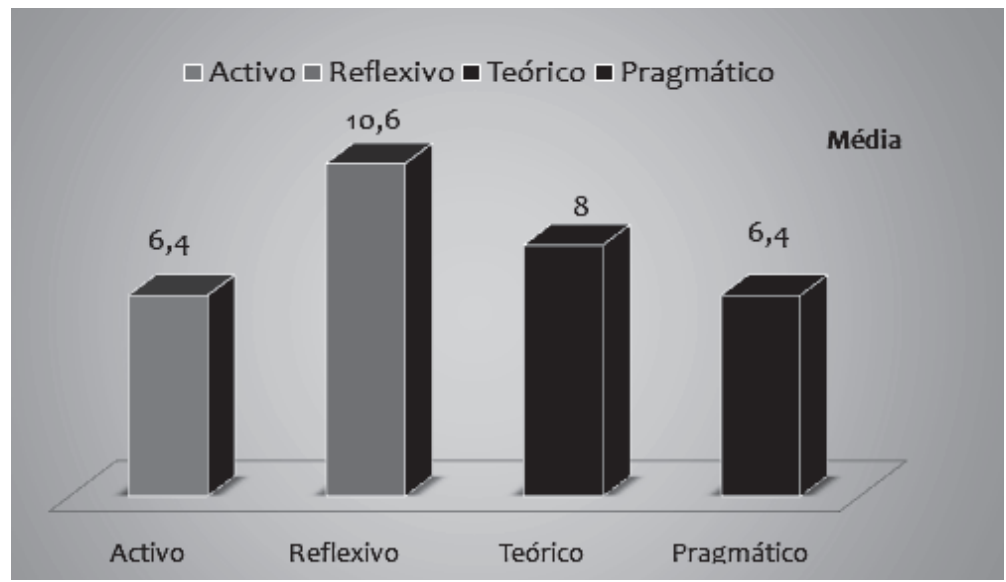

Gráfico 1. Classificação do número de estudantes de acordo com os 4 estilos de aprendizagem

Os resultados da ficha de diagnóstico dos conhecimentos dos estudantes no momento 1 (Gráfico2) revelaram que, 12 estudantes acertaram em 10 respostas, 7 estudantes acertaram 9 e apenas 2 estudantes conseguiram responder correctamente a 14 respostas. $\mathrm{O}$ valor mais baixo de respostas certas foi 7 , valor este obtido por 2 estudantes. A média foi de 10,5 respostas certas para 33 estudantes.

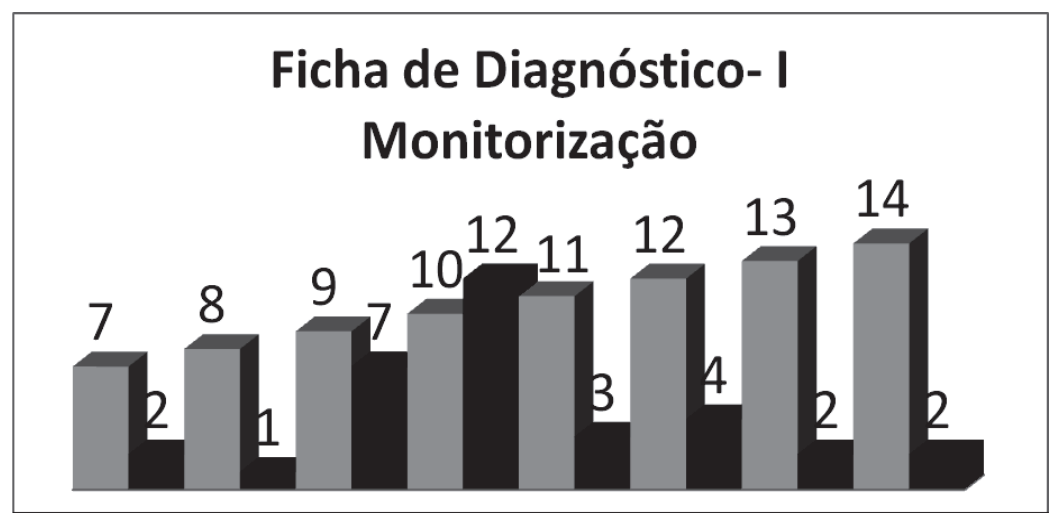

Gráfico 2. Distribuição dos estudantes e número de respostas certas no momento de avaliação1

Com o intervalo de tempo de 45 dias e com 12 sessões concluídas de tipologia teórica, aplicou-se a ficha de avaliação de conhecimentos igual à anterior para se obter a evolução na aquisição dos conhecimentos. 
Os resultados apontaram que para um número de 18 estudantes que preencheram este instrumento (taxa de resposta de 51\%) a média de respostas certas foi 15 (Gráfico 3).

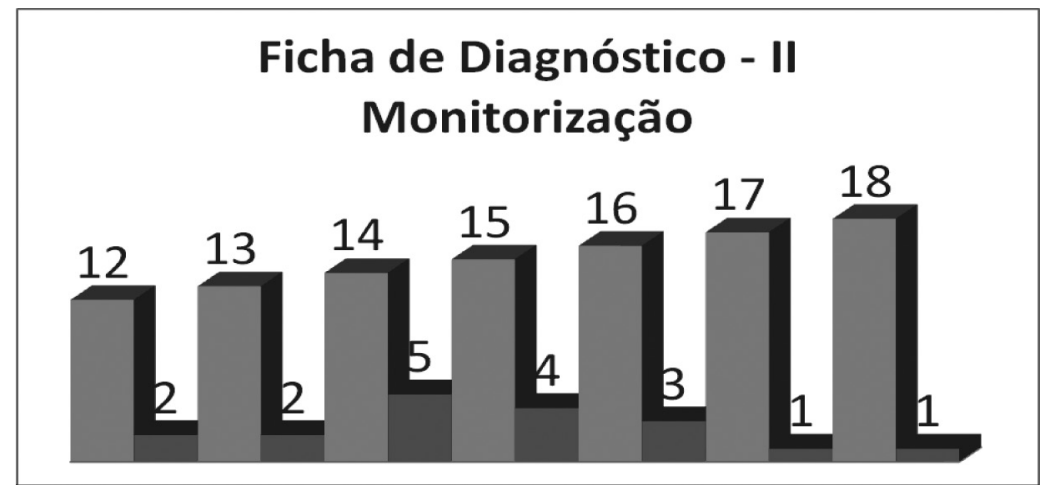

Gráfico 3. Distribuição dos estudantes e número de respostas certas no momento de avaliação 2

Verificou-se que 5 estudantes responderam correctamente a 14 respostas, 4 estudantes acertaram em 16 respostas e um dos estudantes acertou em 18 respostas.

A avaliação da aprendizagem, numa escala entre o e 20 valores revelou os resultados médios apresentados na Figura 9 e representados graficamente no Gráfico 5 . 


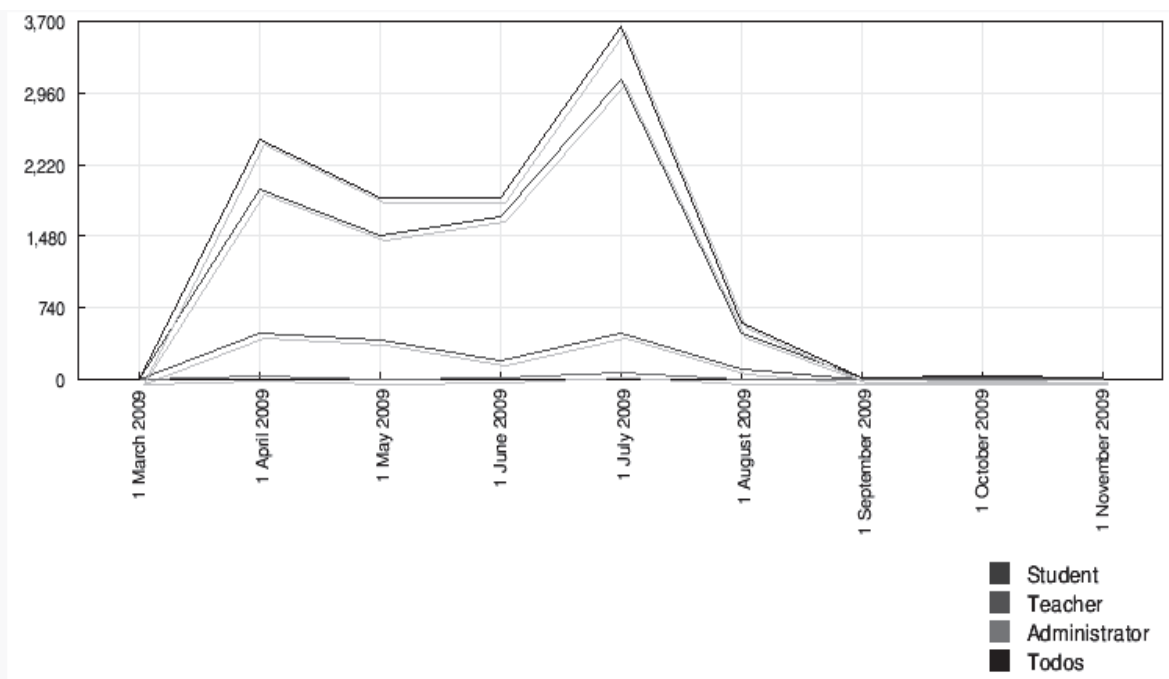

Gráfico 4. Registo da monitorização das actividades na plataforma moodle

O Gráfico 4, apresenta o registo do processo das actividades dos estudantes, docentes e administrador da plataforma. Realça-se que seguido a um pico do docente se segue imediatamente um pico dos estudantes. O período que registou menos actividades, foi entre o final de Abril e princípio do mês de Maio de 2009 pois correspondeu a uma pausa lectiva.

A avaliação constou dos momentos assinalados na tabla 2 e a média final foi de 14,37 valores. Foi aplicado um teste escrito individual dividido em duas partes iguais e de idêntica complexidade, uma utilizando o método presencial e a outra através da plataforma moodle. As actividades de avaliação do ensino através da plataforma moodle que incidiram sobre tópicos programáticos leccionados em metodologia assíncrona e actividades de avaliação complementares registaram, nas classificações, uma diferença média de $+3,01$ para os testes e $+0,47$ para as restantes actividades, com vantagem para a avaliação assíncrona. As actividades de avaliação complementares foram realizadas por via da plataforma moodle e o trabalho escrito em grupo foi apresentado em suporte papel. 


\begin{tabular}{|c|c|c|c|c|c|}
\hline $\begin{array}{c}\text { Teste Parte 1 } \\
\text { (Moodle) }\end{array}$ & $\begin{array}{c}\text { Teste Parte 2 } \\
\text { (Escrito) }\end{array}$ & Total Teste & $\begin{array}{c}\text { Act. } \\
\text { Complement. } \\
\text { Moodle }\end{array}$ & $\begin{array}{c}\text { Trabalho } \\
\text { escrito }\end{array}$ & Final \\
\hline 8,34 & 5,33 & 13,72 & 15,33 & 14,86 & 14,37 \\
\hline
\end{tabular}

Tabla 2. Resultados parcelares e avaliação final da aprendizagem numa escala de o a 20 valores

O Gráfico 5, mostra que a taxa de sucesso foi de $100 \%$ (não houve estudantes reprovados). Apenas um estudante obteve a classificação mínima de 10 valores, valor concordante com as suas restantes classificações.

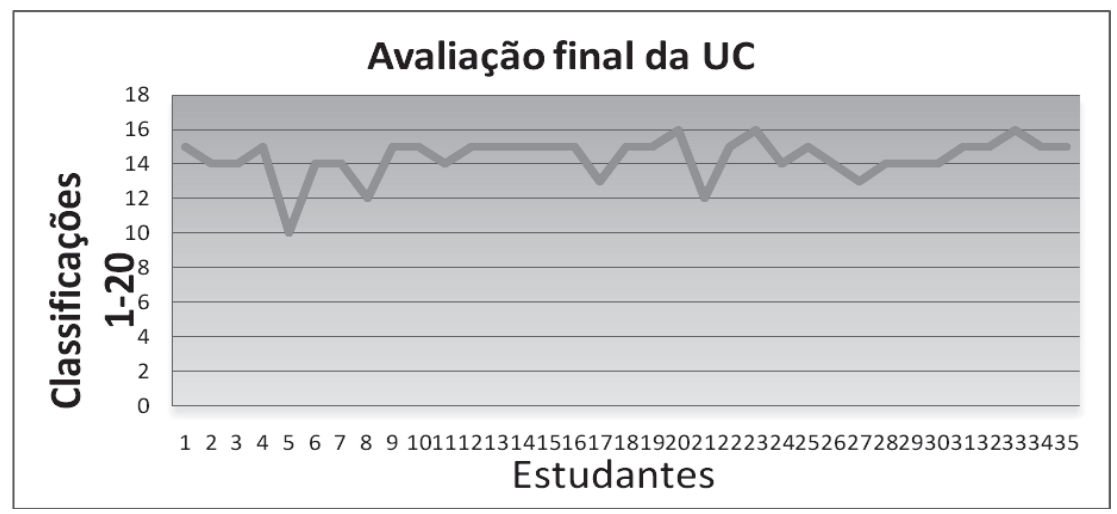

Gráfico 5. Representação das classificações finais [o-20] para os 35 estudantes

Pensa-se, porém, que esse resultado não possa ser atribuído às metodologias e estrutura de leccionação adoptadas pois os resultados para o mesmo estudante noutras UC (s) do plano de estudos, são idênticas.

\section{CONCLUSÕES}

Do ponto de vista da viabilidade e sustentabilidade salienta-se a promoção, de maior contenção nos gastos pela eficiente utilização dos recursos previamente existentes. Relativamente ao plano financeiro pensa-se que uma limitação deste estudo é a não apresentação dos resultados com base nos indicadores de desempenho, demonstrando a sua viabilidade financeira e accountability.

Do ponto de vista científico e pedagógico, salienta-se um impacto global positivo e parece-nos ter conseguido atingir os objectivos propostos. Pode-se verificar, que as 
classificações obtidas na avaliação feita em plataforma moodle e que incidiram sobre os conteúdos leccionados pela mesma plataforma foram superiores.

As vantagens identificadas ajudam a fundamentar a ideia de considerar este tipo de abordagens em situações futuras. No entanto, a falta de alguns recursos para utilização de metodologias híbridas tais como a pouca disponibilidade de terminais para o acesso dos estudantes à plataforma, pode constituir um entrave à adopção deste tipo de soluções. Também a imaturidade tardia que caracteriza as mais recentes gerações de estudantes pode resistir a um tipo de trabalho no qual a decisão é o ponto-chave. Destaca-se também como limitação a inexperiência dos docentes no desenvolvimento de instrumentos em metodologias combinadas. Foram registadas outras limitações à implementação desta metodologia tais como:

Comportamentos de resistência inicial por parte dos estudantes; Ineficiências no acesso à rede informática; Pouca disponibilidade de terminais para o acesso dos estudantes à plataforma dentro da escola; Elevada exigência de tempo e aumento da carga de trabalho para os docentes; A não existência de meios para controlo da identidade do indivíduo que responde ao teste de avaliação em metodologia assíncrona.

Como considerações finais, refere-se que esta metodologia apresenta como características principais o facto de ser centrada no estudante, apresentar problemas concretos em contexto clínico, ser um processo activo, cooperativo, integrado, interdisciplinar e orientado para a responsabilização do estudante.

Permitiu promover uma maximização da aprendizagem, fundamental para aprender a utilizar as melhores estratégias educativas relacionadas com as características dos formandos e as suas principais necessidades. Do mesmo modo, permite-nos fazer face aos constrangimentos de recursos, logísticos, físicos e humanos no contexto educativo do curso Superior de Radiologia da ESTeSL em consequência do aumento crescente de estudantes.

Em complemento com o ensino presencial a adopção de modalidades virtuais pode favorecer o impacto do desenvolvimento de competências transversais e específicas nos estudantes. Os resultados de outros estudos efectuados mostram um aumento da interacção, acesso a conteúdos e serviços, flexibilidade, incremento de resultados de aprendizagem e satisfação global dos estudantes e docentes. 
Espera-se com este projecto incrementar o sucesso dos estudantes de Radiologia da ESTeSL para assim poderem competir em circunstâncias mais favoráveis aos escassos postos de trabalho e às crescentes exigências do sector da prestação de cuidados de saúde em Radiologia.

Aspira-se para a Licenciatura em Radiologia uma efectiva adequação curricular ao novo plano de estudos de Bolonha e reconhecimento pela Agência Nacional de Acreditação bem como pela Área Europeia de Ensino Superior (EHEA). Esta UC ao seguir as Guidelines Europeias e a observância das normas de avaliação externa das agências de avaliação, tenta responder em cada momento aos objectivos de aprendizagem dos estudantes e às especificidades do mercado de trabalho em Radiologia.

Acompanhar o desenvolvimento tecnológico, tirar partido e seguir os exemplos de boas práticas com recurso às novas tecnologias e metodologias de aprendizagem, bem como desenvolver iniciativas e actividades adequadas aos ambientes online autónomos, parece-nos constituir uma tendência de futuro. Devemos ter em conta que a formação dos Técnicos de Radiologia hoje, deve ensinar a pensar e a desenvolver competências relacionadas com as técnicas, métodos e tecnologias para um "fazer" rigoroso. O desenvolver da abordagem humanista do cidadão no seu todo por uma prestação de mais e melhores cuidados de saúde disseminada ao longo do espaço e do tempo é também nosso propósito.

\section{REFERENCIAS BIBLIOGRÁFICAS}

Carvalho, C. V. (org.) (2006). E-learning e formação avançada. Casos de sucesso no Ensino Superior da Europa e América Latina. Porto: Edições Politemo.

Copetto, M. A. (2009). Relatório da OCDE e o Corporativismo. Diário Económico in Ensino Superior hoje. Lisboa: Análise educativa.

Correia, C.; Tomé, I. (2007). O que é o e-learning. Lisboa: Plátano Editora.

Delisle, R. (2000). Como realizar a aprendizagem baseada em problemas. 13 ASA editores II. Porto: Cadernos do CRIAP.

INA, IP. Formação em e-Learning $e$ comunicação. [en línea] Disponible en: http://elearning.ina.pt (consulta 2008, 12 de septiembre).
INA. (2008). Compendium of good practices. Cases of e-learning. Editor Danish Technological Institute.

IPL/UL. Proposta de critérios a observar pela Unidades Orgânicas do Instituto Politécnico de Lisboa tendo em vista a sua adesão ao processo de integração do IPL na UL (2006). Documento de trabalho.

MCTES. (2007). Um compromisso com a Ciência para o Futuro de Portugal vencer a atraso científico e tecnológico. Ministério da Ciência Tecnologia e do Ensino Superior. [en línea] Disponible en: http://www.mctes.pt/archive/doc/ MCTES compromisso com a ciencia. pdf (1-12). (consulta 2008, 18 de agosto). 
Mendes, I.; Dias, A. (2004). Uma experiência de b-learning no âmbito de uma disciplina de licenciatura da Universidade do Minho. In: Universidade do Minho, Braga. [en línea] Disponible en: http://www. sapia.uminho.pt/uploads/uma\%20 experiencia\%2ob-learning.pdf (consulta 2009, 14 de noviembre).

Ministers for Higher Education (2009). Statement by the Bologna Policy Forum Benelux MCTES. [en línea] Disponible en: http://www.mctes.pt/ archive/doc/Bologna Policy Forum Statement 29April2009.pdf (consulta 2009, enero).

Morais N. S.; Cabrita, I. (2007). Ambientes Virtuais de aprendizagem no ensino superior: Comunicação (as) síncrona e Interacção. In actas do IX Simpósio Internacional de Informática Educativa. Porto. versão CD-ROM (223-228).

Morais. N. S.; Cabrita, I. (2008). B-Learning: impacto no desenvolvimento de competências no ensino superior politécnico. Barcelos, Tékhne., i-9 (194-224). [en línea] Disponible en: http://www.scielo.oces.mctes.pt/scielo. php? script $=$ sci arttext\&pid $=S 1654-$ 99112008000100010\&lng $=\mathrm{pt} \& \mathrm{nrm}=\mathrm{iso}$ (consulta 2009, 1 de agosto).
Organization for Economic Co-operation and Development-OECD (2005). E-learning in Tertiary Education: Where do We Stand?. Editor Policy Brief. (1-8).

Organization for Economic Co-operation and Development -OECD (2009). Education Today: the OECD Perspective - Centre for educational research and Innovation. Versão e-book (pdf format) ISBN 46 978-92-64-059955.

Portugal, MCTES, Lei $\mathrm{n}^{\circ} .52$ publicada no DR. No .169 Série I de 2006-09-01.

Press Statement (2008). The OCDE Experts Panel Report. [en línea] Disponible en: http://www.mctes.pt/archive/ doc/Press Statement 07 03 08.pdf (consulta 2009, 12 de septiembre).

Benelux (2009). The Bologna Process 2020 - The European Higher Education Area in the new decade. Communiqué of the Conference of European Ministers Responsible for Higher Education, Leuven and Louvain-la-Neuve, 2829 April 2009. [en línea] Disponible en: http://www.mctes.pt/archive/ doc/Leuven Louvain-la-Neuve Communique April 2009.pdf(consulta 2009, 12 de septiembre). 


\section{PERFIL ACADÉMICO Y PROFESIONAL DE LAS AUTORAS}

Maria Margarida do Carmo Pinto Ribeiro. Mestre em Gestão de Serviços de Saúde e Doutoranda em Ciências da Vida na Faculdade de Ciências Médicas da Universidade Nova de Lisboa; Docente na Área Científica de Radiologia da Escola Superior de Tecnologia da Saúde de Lisboa. Portugal.

E-mail: margarida.ribeiro@estesl.ipl.pt

DIRECCIÓN DE LA AUTORA:

Escola Superior de Tecnologia da Saúde de Lisboa

$A v^{\mathrm{a}}$. D. João II, lote 4.69.01

1900 - 098 Lisboa

Filomena Isabel Gonçalves Batalha. Licenciada em Radiologia e Técnica de Radiologia Especialista no Centro Hospitalar Lisboa Central - Hospital de Santa Marta. Mestranda em Supervisão Pedagógica - Universidade Aberta. Lisboa. Portugal.

E-mail: filobatalha@hotmail.com

DIRECCIÓN DE LA AUTORA:

Rua Das Musas, Parque das Nações, Lote 2.05.04 $1^{\circ} \mathrm{B}$ 1990 - 174 Lisboa

Rosa Joana dos Santos Patrocínio. Licenciada em Radiologia e Técnica de Radiologia no Hospital de Curry Cabral em Lisboa. Tutora em prática clínica dos estudantes do $4^{\circ}$ ano do Curso da Licenciatura em Radiologia. Lisboa. Portugal.

E-mail: patrocioniojoana@sapo.pt

DIRECCIÓN DE LA AUTORA:

Hospital de Curry Cabral - Serviço de Imagiologia

Rua da Beneficência No 8

1069-639 Lisboa

Fecha de recepción del artículo: 25/09/10

Fecha de aceptación del artículo: 14/12/10 